\title{
REVIEW
}

\section{Review of Post-Marketing Safety Data on Tapentadol, a Centrally Acting Analgesic}

\author{
Ariane Stollenwerk · Melanie Sohns · Fabian Heisig • \\ Christian Elling · Detlef von Zabern
}

Received: October 13, 2017 / Published online: December 21, 2017

(C) The Author(s) 2017. This article is an open access publication

\begin{abstract}
Introduction: Tapentadol is a centrally acting analgesic that has been available for the management of acute and chronic pain in routine clinical practice since 2009.

Methods: This is the first integrated descriptive analysis of post-marketing safety data following the use of tapentadol in a broad range of pain conditions relating to the topics overall safety, dose administration above approved dosages, administration during pregnancy, serotonin syndrome, respiratory depression, and convulsion. The data analyzed pertain to spontaneous reports from healthcare and non-healthcare professionals and were put in the context of safety information known from interventional and non-interventional trials.

Results: The first years of routine clinical practice experience with tapentadol have confirmed the tolerability profile that emerged from the
\end{abstract}

Enhanced content To view enhanced content for this article go to http://www.medengine.com/Redeem/ CDFCF06013CC81E4.

Electronic supplementary material The online version of this article (https://doi.org/10.1007/s12325017-0654-0) contains supplementary material, which is available to authorized users.

A. Stollenwerk $(\bowtie) \cdot$ M. Sohns · F. Heisig .

C. Elling · D. von Zabern

Grünenthal GmbH, Aachen, Germany

e-mail: ariane.stollenwerk@grunenthal.com clinical trials. Moreover, the reporting of expected side effects such as respiratory depression and convulsion was low and no major risks were identified. The evaluation of available post-marketing data did not confirm the theoretical risk of serotonin syndrome nor did it reveal unexpected side effects with administration of higher than recommended doses.

Conclusion: More than 8 years after its first introduction, the favorable overall safety profile of tapentadol in the treatment of various pain conditions is maintained in the general population.

Funding: Grünenthal GmbH.

Keywords: Acute pain; Analgesia; Chronic pain; Pain; Routine clinical practice; Safety profile; Tapentadol; Tolerability

\section{INTRODUCTION}

Tapentadol, a centrally acting analgesic, has been proposed as the first agent in a new class of drugs (MOR-NRI [1]) combining the two mechanisms of action $\mu$-opioid receptor (MOR) agonism and noradrenaline reuptake inhibition (NRI) in one molecule [2]. MOR activation in tapentadol is considerably lower compared to classical opioids; however, the synergistic interaction of the two mechanisms allows for a strong analgesic effect and likely results in fewer 
opioid-typical side effects $[2,3]$. Tapentadol can be considered a "clean" molecule, exerting its analgesic effects directly and having no active metabolites [4]; the main metabolic pathway is glucuronidation [5]. The potential for pharmacokinetic drug-drug interaction is low; clinically relevant interactions are unlikely to occur $[6,7]$.

Tapentadol is available as immediate release (IR) film-coated tablets (dose strengths 25, 50, $75,100 \mathrm{mg}$ ), oral solution (4 and $20 \mathrm{mg} / \mathrm{ml}$ ), and as prolonged release (PR) film-coated tablets (dose strengths 25, 50, 100, 150, 200, $250 \mathrm{mg}$ ). It is indicated in Europe "for the relief of moderate to severe acute pain in adults, which can be adequately managed only with opioid analgesics" (IR formulation/oral solution) and "for the management of severe chronic pain in adults, which can be adequately managed only with opioid analgesics" (PR formulation). In the USA, tapentadol is approved "for the relief of moderate to severe acute pain in patients 18 years of age or older" (IR formulation/oral solution) and "for the management of moderate to severe chronic pain in adults when a continuous, around-the-clock opioid analgesic is needed for an extended period of time" (PR formulation). In addition, the PR formulation is indicated in the USA for the management of painful diabetic peripheral neuropathy. A pediatric development program is currently ongoing both in Europe and the USA.

Both the IR and PR formulations demonstrated efficacy and tolerability in a broad range of pain conditions [8,9]. Phase 2 and 3 clinical trials showed effective analgesia of tapentadol IR in moderate to severe acute postoperative pain [10-12] and osteoarthritis or low back pain $[13,14]$. Tapentadol PR was proven effective in randomized clinical trials for the management of moderate to severe chronic pain conditions such as osteoarthritis pain $[15,16]$, low back pain [16-19], painful diabetic peripheral neuropathy $[20,21]$, and cancer pain $[22,23]$. Longterm pain relief under tapentadol PR (for up to 2 years) could be safely maintained in the treatment of chronic osteoarthritis or low back pain without any signs of tolerance development [24].
Tapentadol has been available for the management of chronic pain in routine clinical practice in the USA since 2009 and in Germany as the first European country since October 2010; approval in Europe was prolonged with unlimited validity based on the available comprehensive body of evidence in 2015. Tapentadol is also currently approved in Latin America, Asia, Australia, Israel, Japan, and North America, in total in 56 countries worldwide. Recently, a panel of pain specialists reviewed the clinical trial program and discussed the role of tapentadol PR in routine clinical practice management of chronic pain [9]. Apart from effective pain relief and improvements of functionality, health status, and quality of life of the patients, they stated a good safety profile for tapentadol PR, in particular, a favorable gastrointestinal tolerability profile.

The present article provides a comprehensive review of substance-specific safety data collected in the Grünenthal global safety database relating to the topics overall safety, dose administration above approved dosages, administration during pregnancy, serotonin syndrome, respiratory depression, and convulsion. The topics were selected on the basis of their relevance for both patients and prescribers.

\section{METHODS}

\section{Databases}

The Grünenthal global safety database until May 20, 2016 contained 10,758 cases concerning tapentadol. Out of these, all 8199 spontaneous reports from healthcare and nonhealthcare professionals and all 105 spontaneous reports obtained from the literature were included in this review (the remaining 2454 cases originated from solicited data sources such as clinical trials). The objective was to review all spontaneous reports related to overall safety, dose administration above approved dosages, administration during pregnancy, serotonin syndrome, respiratory depression, and convulsions, and to compare these data to relevant clinical trial data if feasible. Clinical trial data 
were obtained from the Grünenthal interventional and non-interventional trial databases. The interventional clinical trial (ICT) database included 49 prospective phase 2,3 , and 4 trials investigating tapentadol IR or PR; phase 1 (healthy volunteers) and pediatric trials were excluded. The database contained 12,506 adult subjects. The non-interventional trial (NIT) database included the first four observational trials assessing tapentadol PR in routine clinical practice in Germany and contained data of 10,689 adult patients. All cases of adverse drug reactions (ADRs) to tapentadol were retrieved from both the ICT and NIT database.

\section{Search Strategies}

Adverse events in all three databases had been encoded with the Medical Dictionary for Regulatory Activities (MedDRA) versions 15.0 and later. All spontaneous report data and clinical trial data were searched using either standardized MedDRA queries (SMQs) or customized MedDRA queries (CMQs).

\section{Dose Administration Above Approved Dosages} Databases were searched using the CMQ "overdose" (included MedDRA preferred term codes are listed in the online supplementary material).

\section{Pregnancy}

Databases were searched using the CMQ "pregnancy and lactation" consisting of the SMQs "fetal disorders", "normal pregnancy conditions and outcomes", "lactation-related topics" (including neonatal exposure through breast milk), "pregnancy, labor, and delivery complications and risk factors" (excluding abortions and stillbirth), "neonatal disorders", and "termination of pregnancy and risk of abortion". The ICT database was also checked for positive pregnancy tests.

\section{Serotonin Syndrome}

Databases were searched using the MedDRA preferred term "serotonin syndrome".

\section{Respiratory Depression}

Databases were searched using the SMQ "acute central respiratory depression" (included MedDRA preferred term codes are listed in the online supplementary material).

\section{Convulsion}

Databases were searched using the SMQ "convulsion" (included MedDRA preferred term codes are listed in the online supplementary material).

\section{Compliance with Ethics Guidelines}

This article is based on previously conducted studies and does not involve any new studies of human or animal subjects performed by any of the authors.

\section{RESULTS}

\section{Overall Safety}

Table 1 shows the demographic data for all spontaneous report cases and all clinical trial cases documenting ADRs. A total of 7185 subjects $(57.5 \%$ of the 12,506 subjects included in the ICT database) and 777 patients (7.3\% of the 10,689 patients included in the NIT database) experienced ADRs.

The most frequent ADRs reported spontaneously and in clinical trials are listed in Table 2. A total of 18,028 ADRs were documented in the 8304 spontaneous reports, 21,312 ADRs were reported for 7185 subjects in ICTs, and 1659 ADRs for 777 patients in NITs. In all databases, gastrointestinal and CNS-related drug reactions were most common; in addition, $6.9 \%$ of the spontaneously reported cases cited "drug ineffective" as an ADR. In clinical trials, however, drug ineffectiveness is already included in efficacy analyses and not collected as ADR; "drug ineffective" is therefore not part of the ADR analysis from clinical trials provided here.

Stratification by age ( $\geq 65$ and $<65$ years) is shown in Table 2 . Nausea and dizziness were most frequently documented for both age 
Table 1 Demographic profile of all included cases

\begin{tabular}{|c|c|c|c|}
\hline & $\begin{array}{l}\text { Spontaneous reports } \\
(n=8304)\end{array}$ & $\begin{array}{l}\text { Interventional clinical trial data } \\
(n=7185)\end{array}$ & $\begin{array}{l}\text { Non-interventional trial data } \\
(n=777)\end{array}$ \\
\hline \multicolumn{4}{|l|}{ Gender } \\
\hline Female & $4340(52.3 \%)$ & $4731(65.8 \%)$ & $529(68.1 \%)$ \\
\hline Male & $2562(30.9 \%)$ & $2454(34.2 \%)$ & $247(31.8 \%)$ \\
\hline $\begin{array}{l}\text { No information } \\
\text { available }\end{array}$ & $1402(16.9 \%)$ & 0 & $1(0.1 \%)$ \\
\hline \multicolumn{4}{|l|}{ Age (years) } \\
\hline $0-9$ & $1(<0.1 \%)$ & 0 & $\mathrm{~N} / \mathrm{A}$ \\
\hline $10-19$ & $31(0.4 \%)$ & $64(0.9 \%)$ & 0 \\
\hline $20-29$ & $184(2.2 \%)$ & $685(9.5 \%)$ & $8(1 \%)$ \\
\hline $30-39$ & $414(5.0 \%)$ & $558(7.8 \%)$ & $29(3.7 \%)$ \\
\hline $40-49$ & 709 (8.5\%) & 1299 (18.1\%) & $81(10.4 \%)$ \\
\hline $50-59$ & $853(10.3 \%)$ & $1947(27.1 \%)$ & $150(19.3 \%)$ \\
\hline $60-69$ & 719 (8.7\%) & $1606(22.4 \%)$ & $143(18.4 \%)$ \\
\hline $70-79$ & $537(6.5 \%)$ & 867 (12.1\%) & $239(30.8 \%)$ \\
\hline $80-89$ & $333(4.0 \%)$ & $155(2.2 \%)$ & $114(14.7 \%)$ \\
\hline $90-99$ & $48(0.6 \%)$ & $4(<0.1 \%)$ & $10(1.3 \%)$ \\
\hline $100-109$ & $1(<0.1 \%)$ & 0 & 0 \\
\hline $\begin{array}{l}\text { No information } \\
\text { available }\end{array}$ & 4474 (53.9\%) & 0 & $3(0.4 \%)$ \\
\hline
\end{tabular}

Data are number of patients (\%)

$N / A$ not applicable

groups. Occurrences of nausea were higher in the elderly according to post-marketing data [spontaneous reports (5.6\% vs. $4.3 \%)$ and NIT database $(17.2 \%$ vs. $15 \%)]$ but lower than for subjects $<65$ years in the ICT database $(12.3 \%$ vs. $17.3 \%$ of all ADRs in this age group). Dizziness was more often reported in the elderly in spontaneous reports $(5.6 \%$ vs. $3 \%)$, occurred more often in younger subjects in the ICT dataset $(11.2 \%$ vs. $10.4 \%)$, and in similar proportions in both groups in the NIT trials $(8.7 \%$ vs. $8.5 \%)$. Vomiting was experienced more frequently in the younger age group in the ICT database $(8.5 \%$ vs. $6.1 \%)$. In all three databases, constipation was more prominent in the elderly
( $2.3 \%$ vs. $1 \%$ for spontaneous reports, $3.8 \%$ vs. $2.2 \%$ in the NIT database, and $10.7 \%$ vs. $5.9 \%$ in the ICT database), whereas headache was more often reported in the younger age group $(2.7 \%$ vs. $1.5 \%$ for spontaneous reports, $4.4 \%$ vs. $2.7 \%$ in the NIT database, and $5.8 \%$ vs. $3.2 \%$ in the ICT database). Confusional state occurred more frequently in the elderly in spontaneous reports ( $3.1 \%$ vs. $1 \%)$.

In summary, the most common side effects of tapentadol were typical side effects under opioid treatment such as nausea; noradrenergic side effects did not seem to be prominent. No new major risks were identified after the product launch; the overall safety profile remains 
Table 2 Most frequently reported adverse drug reactions (MedDRA preferred terms)

\begin{tabular}{|c|c|c|c|}
\hline & All patients & Elderly patients ( $\geq 65$ years) & Patients $<65$ years \\
\hline \multicolumn{4}{|c|}{ Spontaneous reports (global database) } \\
\hline All adverse drug reactions & $18,028(100 \%)$ & $3569(100 \%)$ & $8130(100 \%)$ \\
\hline Drug ineffective & $1237(6.9 \%)$ & $181(5.1 \%)$ & $492(6.1 \%)$ \\
\hline Nausea & $888(4.9 \%)$ & $198(5.6 \%)$ & $346(4.3 \%)$ \\
\hline Dizziness & $644(3.6 \%)$ & $177(5.6 \%)$ & $245(3 \%)$ \\
\hline Headache & $472(2.6 \%)$ & $55(1.5 \%)$ & $222(2.7 \%)$ \\
\hline Hallucination & $336(1.9 \%)$ & $64(1.8 \%)$ & $122(1.5 \%)$ \\
\hline Vomiting & $335(1.9 \%)$ & $81(2.3 \%)$ & $148(1.8 \%)$ \\
\hline Somnolence & $321(1.8 \%)$ & $76(2.1 \%)$ & $148(1.8 \%)$ \\
\hline Feeling abnormal & $297(1.7 \%)$ & $33(0.9 \%)$ & $140(1.7 \%)$ \\
\hline Hyperhidrosis & $248(1.4 \%)$ & $46(1.3 \%)$ & $108(1.3 \%)$ \\
\hline Fatigue & $245(1.4 \%)$ & $53(1.5 \%)$ & $114(1.4 \%)$ \\
\hline Confusional state & $244(1.4 \%)$ & $110(3.1 \%)$ & $81(1 \%)$ \\
\hline Constipation & $228(1.3 \%)$ & $81(2.3 \%)$ & $83(1 \%)$ \\
\hline Dyspnea & $218(1.2 \%)$ & $47(1.3 \%)$ & $114(1.4 \%)$ \\
\hline \multirow[t]{2}{*}{ Pain } & $245(1.4 \%)$ & $44(1.2 \%)$ & $127(1.6 \%)$ \\
\hline & All subjects & Elderly subjects ( $\geq 65$ years) & Subjects $<65$ years \\
\hline \multicolumn{4}{|c|}{ Interventional clinical trial database } \\
\hline All adverse drug reactions & $21,312(100 \%)$ & $5214(100 \%)$ & $16,098(100 \%)$ \\
\hline Nausea & $3429(16.1 \%)$ & $640(12.3 \%)$ & $2789(17.3 \%)$ \\
\hline Dizziness & $2338(11 \%)$ & $542(10.4 \%)$ & $1796(11.2 \%)$ \\
\hline Vomiting & $1689(7.9 \%)$ & $316(6.1 \%)$ & $1373(8.5 \%)$ \\
\hline Somnolence & $1567(7.4 \%)$ & $343(6.6 \%)$ & $1224(7.6 \%)$ \\
\hline Constipation & $1512(7.1 \%)$ & $560(10.7 \%)$ & $952(5.9 \%)$ \\
\hline Headache & $1096(5.1 \%)$ & $169(3.2 \%)$ & $927(5.8 \%)$ \\
\hline Fatigue & $706(3.3 \%)$ & $191(3.7 \%)$ & $515(3.2 \%)$ \\
\hline Dry mouth & $602(2.8 \%)$ & $183(3.5 \%)$ & $419(2.6 \%)$ \\
\hline Pruritus & $549(2.6 \%)$ & $109(2.1 \%)$ & $440(2.7 \%)$ \\
\hline Hyperhidrosis & $432(2 \%)$ & $110(2.1 \%)$ & $322(2 \%)$ \\
\hline Decreased appetite & $300(1.4 \%)$ & $116(2.2 \%)$ & $184(1.1 \%)$ \\
\hline
\end{tabular}


Table 2 continued

\begin{tabular}{lclc}
\hline & All patients & Elderly patients $(\mathbf{2 6 5}$ years $)$ & Patients $<\mathbf{6 5}$ years \\
\hline Non-interventional trial database & & & $773(100 \%)$ \\
All adverse drug reactions & $1659(100 \%)$ & $886(100 \%)$ & $116(15 \%)$ \\
Nausea & $268(16.2 \%)$ & $152(17.2 \%)$ & $66(8.5 \%)$ \\
Dizziness & $143(8.6 \%)$ & $77(8.7 \%)$ & $39(5.1 \%)$ \\
Fatigue & $78(4.7 \%)$ & $39(4.4 \%)$ & $34(4.4 \%)$ \\
Vomiting & $67(4 \%)$ & $33(3.7 \%)$ & $17(2.2 \%)$ \\
Constipation & $51(3.1 \%)$ & $34(3.8 \%)$ & $27(3.5 \%)$ \\
Hyperhidrosis & $50(3 \%)$ & $23(2.6 \%)$ & $21(2.7 \%)$ \\
Somnolence & $49(3 \%)$ & $28(3.2 \%)$ & $34(4.4 \%)$ \\
Headache & $45(2.7 \%)$ & $11(1.2 \%)$ & $20(2.6 \%)$ \\
Diarrhea & $40(2.4 \%)$ & $20(2.3 \%)$ & $17(2.2 \%)$ \\
Restlessness & $40(2.4 \%)$ & $23(2.6 \%)$ & $18(2 \%)$ \\
Abdominal pain upper & $28(1.7 \%)$ & $(1.3 \%)$ &
\end{tabular}

Data are number of adverse drug reactions (\%). Information about age was not available for all reports

unchanged. Overall, tapentadol was well tolerated by adult patients including the elderly.

\section{Dose Administration Above Approved Dosages}

Recommendations for upper dosage limits of the two oral tapentadol formulations are based on the dose range studied during clinical development, i.e., on a lack of study data for higher dosages and not on evidence for a risk associated with higher dosages. Daily doses greater than $700 \mathrm{mg}$ on the first day of treatment and daily maintenance doses greater than $600 \mathrm{mg}$ for tapentadol IR and greater than $500 \mathrm{mg}$ for tapentadol PR have not been studied and are therefore not recommended $[25,26]$. Unlike with tapentadol, maximum recommended dosages are often not included in the summary of product characteristics of classical opioids. Initial and maintenance doses of opioids depend on the patient's pain severity and previous analgesic treatment. Higher than recommended tapentadol dosages have been administered both in the therapeutic setting and in abuse situations which is to be expected for a medication with MOR activity.

\section{Preclinical Data}

Administration of high doses of tapentadol resulted in dose-dependent, predominantly CNS-related, reversible clinical signs such as fearfulness, sedation or excited behavior, recumbency and hunched posture, impaired respiratory function, convulsions (rarely), and, in dogs in particular, salivation, vomiting, and retching. These exaggerated pharmacodynamic effects limited the exposure margins of tapentadol in healthy animals. The $\mathrm{LD}_{50}$ ranged from approx. $350 \mathrm{mg} / \mathrm{kg}$ in mice to greater than $1000 \mathrm{mg} / \mathrm{kg}$ in rats after p.o. administration and was approx. $45 \mathrm{mg} / \mathrm{kg}$ in both species after i.v. administration [27].

\section{Search Results}

Clinical Trial Data The ICT database contained three cases of tapentadol doses above the recommended daily intake leading to adverse events (Table 3 ). 
In routine clinical practice, 48 patients ( $0.45 \%$ of all patients in the NIT database) received a daily dose of tapentadol PR greater than $500 \mathrm{mg}$ at least once during treatment. A daily dose of greater than $750 \mathrm{mg}$ was reported four times; the highest dosage was $875 \mathrm{mg}$ tapentadol PR/day. Ten patients (20.8\%) reported 19 ADRs, six of them severe. Three ADRs were listed as prescribed overdose, the others were restless legs syndrome, initial insomnia, insomnia, memory impairment, diarrhea, hyperhidrosis, withdrawal syndrome (two cases), disturbance in attention, dizziness, nausea, depression suicidal, abdominal distension, abdominal discomfort, head discomfort, and flushing. Recovery was documented for $52.6 \%$ and outcome was not known for the remainder.

Spontaneous Reports in Safety Database A total of 208 cases were retrieved from the global database. In 17 cases, toxicity was reported for dosages within the recommended range; in five of these cases, the event was attributed to other medications. Figure 1 shows the dosage range for the other 191 cases. The highest documented daily tapentadol doses were $2050 \mathrm{mg}$ /day (outcome: recovering from hallucinations, no other events reported) and $5000 \mathrm{mg}$ for a single intake (outcome: recovered). In $47.5 \%$ of the 208 cases ADRs were reported (no ADRs in $37.5 \%$ and unknown in $14.9 \%$ of the cases). Table 4 lists the most frequently reported ADRs.

Sixteen fatalities were reported under tapentadol, two of those with a dosage in the recommended range. In 10 of these cases, the provided information indicates that the patient administered tapentadol together with other drugs or illicit substances; this precludes a clear assessment of the contribution of each substance to the fatal course. Of the remaining six cases, three were cancer patients who likely died from their underlying disease, two cases did not provide any information regarding the circumstances or cause of death, and one case reported on a patient who apparently died from septic shock. Overall, there is not one spontaneously reported case where a fatal outcome could be clearly attributed to a tapentadol intake exceeding the recommended dosage.

Table 3 Adverse events following tapentadol doses above the recommended daily intake (ICT database)

\begin{tabular}{|c|c|c|c|}
\hline & Case 1 & Case 2 & Case 3 \\
\hline Gender & Female & Male & Female \\
\hline $\begin{array}{l}\text { Treatment } \\
\text { indication }\end{array}$ & Chronic low back pain & Osteoarthritis knee pain & Acute pain \\
\hline $\begin{array}{l}\text { Tapentadol } \\
\text { dosage }\end{array}$ & $\begin{array}{l}14,250 \mathrm{mg} \text { tapentadol PR over } \\
10 \text { days }\end{array}$ & $1650 \mathrm{mg}$ tapentadol IR over 13 days & $\begin{array}{l}8475 \mathrm{mg} \\
\text { tapentadol IR } \\
\text { over } 11 \text { days }\end{array}$ \\
\hline $\begin{array}{l}\text { Concomitant } \\
\text { medication }\end{array}$ & No & $\begin{array}{l}\text { No but drug screen was positive for methadone, } \\
\text { benzodiazepines, opiates, and tricyclic } \\
\text { antidepressants }\end{array}$ & Yes \\
\hline Adverse event & $\begin{array}{l}\text { Severe visual disturbance and } \\
\text { euphoria (serious adverse } \\
\text { event) }\end{array}$ & $\begin{array}{l}\text { Severe inadvertent multi-drug overdose with } \\
\text { moderate somnolence and dysarthria }\end{array}$ & $\mathrm{No}^{\mathrm{a}}$ \\
\hline $\begin{array}{l}\text { Relationship } \\
\text { to } \\
\text { tapentadol }\end{array}$ & Probably/likely & Probably/likely & Not related \\
\hline
\end{tabular}

${ }^{a}$ Subject experienced moderate atrial flutter 9 days later which was possibly related; dose at onset was $1800 \mathrm{mg}$ 


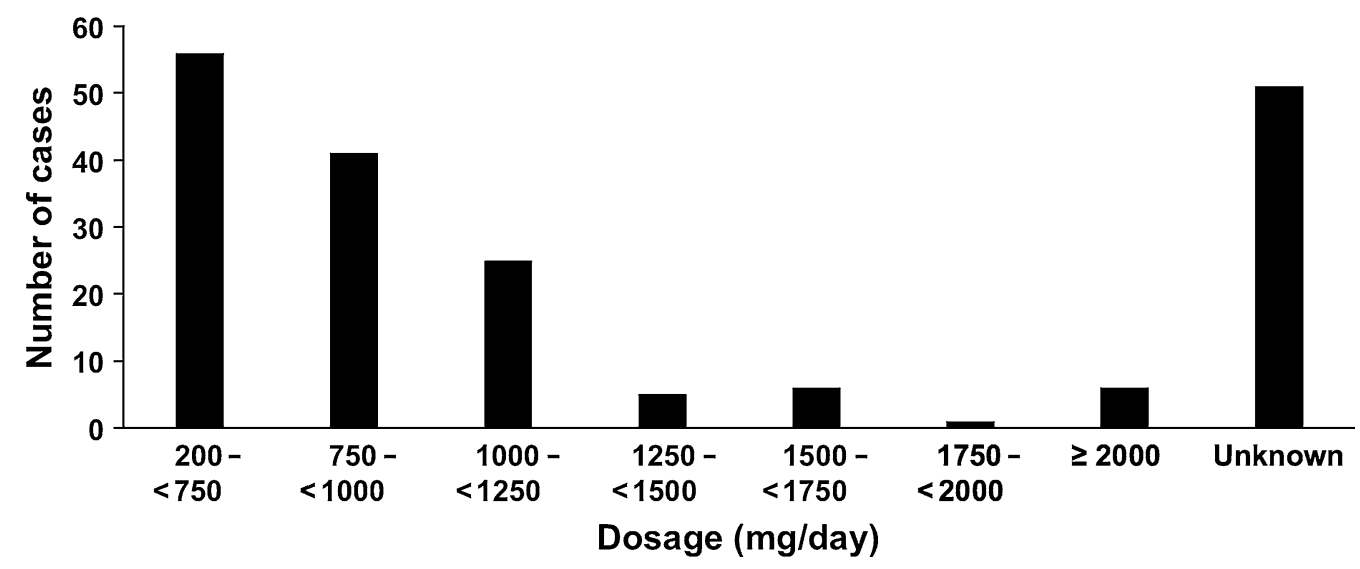

Fig. 1 Dose administration above the recommended dosage (either the total daily dose or a single administered dose; $n=191 ;$ spontaneous reports)

Table 4 Most common adverse drug reactions (MedDRA system organ class and preferred term) with tapentadol intake exceeding the recommended dosage (spontaneous reports)

\begin{tabular}{lc}
\hline All adverse drug reactions & $\mathbf{4 5 8}(\mathbf{1 0 0 \% )}$ \\
\hline General disorders and administration site conditions \\
Drug ineffective & $35(7.6 \%)$ \\
Psychiatric disorders & $12(2.6 \%)$ \\
Withdrawal syndrome & $11(2.4 \%)$ \\
Hallucination & $10(2.2 \%)$ \\
Suicide attempt & $9(2.0 \%)$ \\
Drug abuse & $10(2.2 \%)$ \\
Nervous system disorders & $9(2.0 \%)$ \\
Unresponsive to stimuli & $15(3.3 \%)$ \\
Somnolence & $9(2.0 \%)$ \\
Injury, poisoning, and procedural complications \\
Toxicity to various agents \\
Respiratory, thoracic, and mediastinal disorders \\
Respiratory depression
\end{tabular}

Data are number of adverse drug reactions (\%)

A manual case review showed that ADR incidences differed according to dosage intake (Table 5; this information is not captured in the structured fields of our database). Fifty-one cases
Table 5 Most common adverse drug reactions (MedDRA preferred term) according to tapentadol dosage (spontaneous reports)

\begin{tabular}{|c|c|c|}
\hline & $\begin{array}{l}500-<1000 \mathrm{mg} \\
(n=97)\end{array}$ & $\begin{array}{l}\geq 1000 \mathrm{mg} \\
(n=43)\end{array}$ \\
\hline Drug ineffective & $22(22.7 \%)$ & $6(14 \%)$ \\
\hline Hallucination & $7(7.2 \%)$ & $1(2.3 \%)$ \\
\hline Suicide attempt & $7(7.2 \%)$ & $2(4.7 \%)$ \\
\hline Constipation & $6(6.2 \%)$ & 0 \\
\hline $\begin{array}{l}\text { Withdrawal } \\
\text { syndrome }\end{array}$ & $6(6.2 \%)$ & $3(7 \%)$ \\
\hline Confusional state & $5(5.2 \%)$ & $1(2.3 \%)$ \\
\hline Dizziness & $5(5.2 \%)$ & $2(4.7 \%)$ \\
\hline Drug abuse & $5(5.2 \%)$ & $3(7 \%)$ \\
\hline Somnolence & $5(5.2 \%)$ & $1(2.3 \%)$ \\
\hline Dyspnea & 0 & $2(4.7 \%)$ \\
\hline Hypersomnia & 0 & $2(4.7 \%)$ \\
\hline $\begin{array}{l}\text { Respiratory } \\
\text { depression }\end{array}$ & $2(4.7 \%)$ & $2(4.7 \%)$ \\
\hline $\begin{array}{l}\text { Unresponsive to } \\
\text { stimuli }\end{array}$ & $1(2.3 \%)$ & $2(4.7 \%)$ \\
\hline Vomiting & $4(9.3 \%)$ & $2(4.7 \%)$ \\
\hline
\end{tabular}

Data are number of cases 
with unknown dosage and 17 cases with reported dosage in the recommended range were not included in this analysis. Of the 140 included cases 97 reported a dosage of less than $1000 \mathrm{mg}$ and 43 a dosage of at least $1000 \mathrm{mg}$ tapentadol. The 97 cases included 18 (18.6\%) single intakes and $6(6.2 \%)$ abuse/misuse cases; tapentadol was administered as pain medication in 51 cases $(52.6 \%)$. A multiple drug overdose was reported in 8 cases $(8.2 \%)$. ADRs occurred in 46 cases $(47.4 \%)$. The 43 cases included 12 (27.9\%) single intakes and 3 (7\%) abuse/misuse cases; tapentadol was administered as pain medication in 16 cases $(37.2 \%)$. A multiple drug overdose was reported in 2 cases (4.7\%). ADRs occurred in 20 cases (46.5\%).

In summary, administration of higher than recommended tapentadol doses was not associated with side effects in a third of the cases; furthermore, some of the side effects reported are also expected for therapeutic dosages. Many of the reported cases occurred during pain treatment in order to achieve stronger analgesia; in some cases, abuse and suicide attempts were the reason for a higher tapentadol intake, mostly in combination with other medications. The reporting rate of fatalities with tapentadol alone was low; these fatalities were mostly associated with the underlying disease and not with the use of tapentadol.

\section{Pregnancy}

Exposure to opioids in utero can lead to neonatal opioid withdrawal symptoms [neonatal abstinence syndrome (NAS)]. The absolute risk following prenatal exposure to prescription opioids was low in the absence of additional risk factors; long-term use and use in late pregnancy increased the risk independent of additional risk factors [28]. Opioids in early pregnancy may also be associated with birth defects such as congenital heart defects, neural tube defects, and clubfoot [29]. Owing to the MOR activity of tapentadol, the risk of respiratory depression for neonates also needs to be considered.

First trimester pregnancy exposure to serotonin-noradrenaline reuptake inhibitors (SNRIs) such as venlafaxine and duloxetine which-like tapentadol-include the NRI mechanism of action have been reviewed for major congenital malformations [30]; there was no increased risk with venlafaxine treatment and no clinically important risk with duloxetine. Venlafaxine was also judged as relatively safe during pregnancy (relating to major malformations) but the authors could not draw a definite conclusion regarding breastfeeding [31].

\section{Preclinical Data}

Tapentadol was not genotoxic in bacteria in the Ames test. Equivocal findings were observed in an in vitro chromosomal aberration test, but when the test was repeated the results were clearly negative. Tapentadol was not genotoxic in vivo, using the two endpoints of chromosomal aberration and unscheduled DNA synthesis, when tested up to the maximum tolerated dose. Long-term animal studies did not identify a potential carcinogenic risk relevant to humans [26]. Fertility was not influenced in male and female rats but there was reduced in utero survival at high doses [26]. Tapentadol showed no teratogenic effects in rats and rabbits following both intravenous and subcutaneous administration. However, delayed development and embryotoxicity were observed for doses resulting in exaggerated pharmacology ( $\mu$-opioid-related CNS effects linked to dosing above the therapeutic range). After intravenous dosing in rats, reduced in utero survival was seen [26].

Tapentadol is excreted in milk; rat pups suckled by dams administered tapentadol were exposed dose-dependently to tapentadol and tapentadol O-glucuronide [26]. Twice daily tapentadol doses which did not provoke maternal toxicity already caused increased mortality of first birth generation (F1) rat pups that were exposed via milk between days 1 and 4 postpartum. There were no effects on neurobehavioral parameters of the F1 generation [26].

\section{Search Results}

Clinical Trial Data This analysis also included clinical data from phase 1 trials in healthy volunteers who received tapentadol. Overall, 14 
Table 6 Outcome of pregnancy in patients exposed to tapentadol

\begin{tabular}{lll}
\hline & $\begin{array}{l}\text { Spontaneous reports (global } \\
\text { database) }\end{array}$ & $\begin{array}{l}\text { Interventional clinical trial } \\
\text { database }\end{array}$ \\
\hline Pregnancies reported & 39 & 14 \\
Outcome unknown & 25 & 3 \\
False positive pregnancy test & 0 & 1 \\
Outcome pending & 5 & 0 \\
Elective termination & 1 & 2 \\
Fetal demise in utero & 0 & 1 \\
Life birth & 8 & 7 \\
Normal term delivery & 6 & 5 \\
Premature birth & 1 & 2 \\
Birth defects & 0 & 0 \\
Neonates experiencing withdrawal & 2 & 0 \\
$\quad$ syndrome & 4 & 0 \\
Exposure via breast milk & 0 & 0 \\
Adverse reactions following breastfeeding & 0 & \\
\hline
\end{tabular}

pregnancies were reported (Table 6); 10 patients were exposed to tapentadol short-term (1-12 days) and four long-term (104-367 days). Outcome was unknown in three cases, one subject had a false positive pregnancy test, and two patients elected to terminate the pregnancy (documentation did not specify the reason but reported no anomaly). Fetal demise in utero approx. 6 weeks after a positive pregnancy test on day 183 of long-term tapentadol PR treatment (last dose $400 \mathrm{mg} /$ day) was documented for one subject.

Seven life births were documented: five term babies (no birth defects or neonatal problems) and two premature births (no anomaly reported). One subject was exposed to tapentadol PR for 367 days (most recent dose $500 \mathrm{mg} /$ day) and delivered a healthy term baby approx. 36 weeks following study completion.

The NIT database did not contain any pregnancy cases.

Spontaneous Reports in Safety Database In total, 39 pregnancies were reported (Table 6).
Outcome was unknown in 25 cases (64.1\%), pending in 5 cases $(12.8 \%) ; 8$ life births $(20.5 \%)$ and one termination were reported. The life birth cases included four term babies (no birth defects or neonatal problems), two term babies with neonates experiencing withdrawal syndrome (mothers had received other opioids concomitantly with tapentadol), one premature birth (no anomaly reported), and one unknown outcome. The termination was elective; documentation did not specify the reason but reported no anomaly.

Exposure to tapentadol via breast milk (i.e., administration to the breastfeeding mother) was reported in four cases (dosage known in one case: $100 \mathrm{mg}$ tapentadol PR b.i.d.), all without adverse drug reactions.

According to the summary of product characteristics [26], tapentadol should be used in pregnancy only if the potential benefit to the mother justifies the potential risk to the fetus. The medication should not be used during breastfeeding. Although our analysis of the clinical and safety databases showed no birth 
defects following exposure to tapentadol and mostly healthy, normal term babies, no reliable conclusions can be drawn because of the low number of cases.

\section{Serotonin Syndrome}

Serotonin syndrome is a spectrum of serotonergic adverse reactions resulting from overactivation of both central and peripheral serotonin receptors due to increased serotonin levels $[32,33]$. The syndrome is characterized by a triad of neuro-excitatory features: neuromuscular hyperactivity, autonomic hyperactivity, and altered mental status $[32,33]$. The most accepted diagnostic criteria are the Hunter serotonin toxicity criteria which are based on the following seven clinical features: clonus, agitation, diaphoresis, tremor, hyperreflexia, hypertonia, and temperature greater than $38^{\circ} \mathrm{C}$ [34]. Serotonin symptoms can result from initiation of monotherapy with a serotonergic substance, dose increases or overdosing with a serotonergic medication, or through interactions of two serotonergic medications acting by different mechanisms [32, 33]. Mechanisms associated with the syndrome are inhibition of serotonin uptake, decreased serotonin metabolism, increased serotonin synthesis, increased serotonin release, activation of serotonergic receptors, and inhibition of certain cytochrome P450 enzymes.

\section{Preclinical Data}

The pharmacological activity of tapentadol results from the synergistic interaction of the two mechanisms MOR agonism and NRI [2]. The inhibitory activity at the noradrenaline reuptake transporter ( $\mathrm{Ki}$ value $0.48 \mu \mathrm{M}$ ) is approx. five times weaker than the binding affinity for the rat MOR (Ki value $0.096 \mu \mathrm{M}$ ) [35] and considerably weaker than a classical potent NRI agent such as desipramine (Ki value $0.001 \mu \mathrm{M})$ [27]. Tapentadol is also only a very weak inhibitor of the 5-HT reuptake transporter in rat brain synaptosomes (Ki value $2.37 \mu \mathrm{M}$ ) compared to the selective serotonin reuptake inhibitor (SSRI) fluoxetine and the SNRIs venlafaxine and duloxetine with Ki values of 0.026 ,
0.062 , and $0.005 \mu \mathrm{M}$, respectively [35]. Furthermore, binding affinity to the human 5-HT reuptake transporter is low ( $\mathrm{Ki}$ value $5.28 \mu \mathrm{M}$ ) compared to fluoxetine, venlafaxine, and duloxetine $(0.002,0.035$, and $0.00035 \mu \mathrm{M}$, respectively [35]). Rat intracerebral microdialysis studies showed a large dose-dependent increase in extracellular hippocampal noradrenaline concentrations (approx. $450 \%$ above baseline at $10 \mathrm{mg} / \mathrm{kg}$ ) and a much smaller increase in extracellular 5-HT concentrations (approx. 130\% above baseline at $10 \mathrm{mg} / \mathrm{kg}$ ) under tapentadol [35]; the reference compounds venlafaxine and duloxetine produced up to sixfold concentration increases for both substances [36].

Using the low-intensity tail flick rat model, the pharmacological activity of tapentadol was compared to the SSRI citalopram and the SNRI reboxetine [37]. The effects of citalopram were inhibited by the serotonin receptor antagonist ritanserin and the effects of reboxetine by the noradrenergic receptor antagonist yohimbine. Tapentadol effects were antagonized by yohimbine but not by ritanserin. This suggests that serotonergic activity is not contributing to the pharmacological activity of tapentadol in this animal model.

Since tapentadol inhibits the 5-HT reuptake transporter only weakly, a clinically relevant influence on 5-HT leading to the development of serotonin syndrome is assessed as to be unlikely.

\section{Search Results}

Clinical Trial Data There were no reports of serotonin syndrome in the ICT database of phase 2,3 , and 4 prospective trials and only one case in the NIT database which is described below.

An adult female participant in one of the NIT trials received $200 \mathrm{mg}$ /day tapentadol PR for the treatment of complex regional pain syndrome and post-traumatic pain and $75 \mathrm{mg}$ /day amitriptyline for neuropathic pain. She experienced nausea, vomiting, pyrexia up to $39^{\circ} \mathrm{C}$, tachycardia, restlessness, and tremor approx. $8 \mathrm{~h}$ after the first administration of tapentadol. The treating physician suspected a serotonin syndrome which was reported as a serious 
adverse event probably related to tapentadol; treatment with tapentadol was discontinued and the patient recovered. However, symptoms do not fulfill the Hunter diagnostics criteria [34] and it is thus questionable if a serotonin syndrome was present.

Spontaneous Reports in Safety Database A total of 151 spontaneous reports were retrieved from the database. None of these cases involved tapentadol dosages above the recommended level. At least one serotonergic drug was co-administered in 109 cases $(72.2 \%) ; 11.9 \%$ of the subjects received two or three serotonergic drugs concomitantly. These included SNRIs (53 cases), SSRIs (46 cases), and tricyclic antidepressants (TCAs; 12 cases). The outcome of $64.9 \%$ of all 151 included cases was unknown or not reported, $34.4 \%$ were documented as recovered or recovering, and $0.7 \%$ were reported as "not recovered".

Only four cases fulfilled the Hunter serotonin toxicity criteria [34] and had thus been robustly diagnosed (Table 7). In all four cases, concomitant serotonergic medication was taken which could also have triggered this reaction without tapentadol administration.

Overall, preclinical findings suggest that a clinically relevant influence of tapentadol on 5-HT leading to the development of serotonin syndrome is unlikely; this has been confirmed in clinical practice with no increased risk identified under tapentadol treatment. Most of the spontaneous reports were not robustly diagnosed, and cases fulfilling the Hunter criteria did not show a definite association of tapentadol with the occurrence of serotonin syndrome.

\section{Respiratory Depression}

Respiratory depression is a major clinical concern in the treatment with centrally acting analgesics because of the potentially fatal consequences. Hypoxic and hypercapnic responses are both affected; effects are dose-dependent and vary according to the mechanism of action of the administered opioid. Respiratory depression is mainly due to activation of $\mu$ - and $\delta$-receptor subtypes and involves respiratory-related neurons in the brainstem $[39,40]$.

\section{Preclinical Data}

Preclinical data have been published by Tzschentke et al. [27]. Spontaneous respiratory frequency was dose-dependently reduced in conscious rats receiving a 4.64-14.7 mg tapentadol/kg i.v. bolus injection. Using plethysmography, respiratory minute volume was reduced by i.v. tapentadol at $18 \mathrm{mg} / \mathrm{kg}$ but not at 2 and $6 \mathrm{mg} / \mathrm{kg}$ in rat. Lower potency than morphine was observed for tapentadol in the

Table 7 Cases of serotonin syndrome (spontaneous reports)

\begin{tabular}{|c|c|c|c|c|}
\hline & Case 1 & Case 2 & Case 3 & Case $4^{a}[38]$ \\
\hline Gender & $\mathrm{F}$ & $\mathrm{F}$ & $\mathrm{F}$ & M \\
\hline $\begin{array}{l}\text { Tapentadol } \\
\text { dosage }\end{array}$ & $200 \mathrm{mg}$ bid & $200 \mathrm{mg}$ tid & $25 \mathrm{mg}$ & unknown \\
\hline $\begin{array}{l}\text { Concomitant } \\
\text { serotonergic } \\
\text { medication }\end{array}$ & Venlafaxine & Amitriptyline & Duloxetine & $\begin{array}{l}\text { Duloxetine, } \\
\text { amitriptyline }{ }^{\mathrm{b}}, \\
\text { naloxone, oxycodone }\end{array}$ \\
\hline $\begin{array}{l}\text { Relationship to } \\
\text { tapentadol }\end{array}$ & $\begin{array}{l}\text { Insufficient } \\
\text { documentation }\end{array}$ & $\begin{array}{l}\text { Questionable because of the } \\
\text { delay of symptoms after } \\
\text { tapentadol initiation }\end{array}$ & $\begin{array}{l}\text { Plausible temporal } \\
\text { relation to combined } \\
\text { use of both } \\
\text { medications }\end{array}$ & $\begin{array}{l}\text { Alternative explanation: } \\
\text { caused by treatment } \\
\text { with amitriptyline }\end{array}$ \\
\hline
\end{tabular}

a Context: suicide attempt

b Self-medicated 
inhibition of $\mathrm{CO}_{2}$-induced stimulation of respiratory frequency and in the increase in arterial $\mathrm{pCO}_{2}$ in rats. Daily tapentadol or morphine treatment resulted in complete tolerance to the respiratory depression effect within 22 days.

\section{Search Results}

Clinical Trial Data The clinical database contained 20 ADRs, none of them serious: "respiratory depression" (12 cases), "hypoventilation" (3), "hypopnea" (2), "breath sounds abnormal" (2), and "respiratory rate decreased" (1). The majority $(85 \%)$ were mild or moderate in intensity and three ADRs were considered severe by the investigator. Dose at onset of event was available for $80 \%$ of the cases and ranged from 50 to $500 \mathrm{mg}$ tapentadol/day. Fifteen cases were resolved; one case was not resolved at the end of the trial. The outcome was unknown in four cases. Three ADRs (severe respiratory depression, two with mild hypoventilation) led to study withdrawal.

The NIT database only contained one case of respiratory depression (moderate intensity) under tapentadol PR treatment (100 mg/day) for an 86-year-old female patient. The ADR was considered possibly related to tapentadol by the treating physician; tapentadol was discontinued and the patient recovered.

Spontaneous Reports in Safety Database A total of 64 spontaneous reports of respiratory depression were retrieved from the database. Nine subjects (14\%) were administered tapentadol dosages above the recommended levels. Twenty-eight subjects (43.8\%) concomitantly received centrally depressant medications [benzodiazepine, Z-drugs (zolpidem, zopiclone, zaleplon), strong opioids, or a combination of those]. The medical history of 11 subjects included risk factors of respiratory depression such as alcohol use, asthma, chronic obstructive pulmonary disease (COPD), sleep apnea, brain neoplasm, or neoplasm close to respiratory organs. Three fatal cases were reported; all patients had concomitantly taken opioid analgesics or benzodiazepines. One of the patients had the additional risk factors obesity, sleep apnea, asthma, and COPD [41]; another was a cancer patient. The third patient had pulmonary edema and a peritonsillar abscess; infectious mononucleosis was stated as cause of death.

Overall, respiratory depression rarely occurred under tapentadol treatment. In clinical trials, courses were mostly mild or moderate without severe outcome. In most spontaneous cases, other confounding factors were present (concomitant CNS medications, risk factors of respiratory depression).

\section{Convulsion}

Convulsions are a known effect of medications with opioid activity. They can be caused by multiple mechanisms; MOR agonists probably inhibit hippocampal gamma-aminobutyric acid release by interneurons leading to excitatory effects [42]. Results from seizure induction in a mice model suggest that inhibition of neuronal monoamine reuptake is not a major contributor [43]. Commonly used opioid analgesics (codeine, dextromethorphan, hydrocodone, hydromorphone, meperidine, (-)-methadone, morphine, oxycodone, D-propoxyphene, and tramadol) induced dose-related seizures in mice at sufficiently high doses [43]. In pain management, convulsions are only observed for most opioids at doses far exceeding requirements for analgesia [42]. However, incidences of first-time idiopathic seizures under opioid analgesic treatment have been reported [44].

\section{Preclinical Data}

Clinical signs of toxicity in acute toxicity studies in rats and mice after i.v. and p.o. administration included hyperexcitability, irregular respiratory activity, and convulsions [27]. In repeat-dose toxicity studies, single convulsion episodes occurred at tapentadol doses which showed distinct toxicity including deaths in dogs and in pregnant rats at severe intravenous materno-toxic doses [45].

\section{Search Results}

Clinical Trial Data There were no reports of convulsion in the ICT database of phase 2, 3, and 4 prospective trials. It should be noted that subjects with a seizure disorder had been 
excluded from clinical trials. Convulsion was also not documented in the NIT database.

Spontaneous Reports in Safety Database A total of 117 spontaneous reports of convulsion were retrieved from the database. There was no confirmed administration of tapentadol dosages exceeding the recommended levels. In one case, an overdose was suspected (dosage unknown); single intake exceeded $150 \mathrm{mg}$ tapentadol in two cases (200 and $250 \mathrm{mg}$ ).

Twenty-three subjects (19.7\%) had a medical history of convulsion risk factors such as alcohol abuse, brain injury/surgery, history of seizures/epilepsy, or a combination of those. Tapentadol was discontinued in 10 of these cases. Five subjects continued with the medication; recovery was reported for two of them. Information about continuation/discontinuation of tapentadol was not available for eight subjects.

Overall, post-marketing tapentadol exposure including administration to subjects with a medical history of convulsion risk factors (excluded in clinical trials) did not identify a risk beyond the existing knowledge. The occurrence of convulsions under tapentadol treatment is low, and this adverse drug reaction has been listed as rare.

\section{DISCUSSION}

This review presents the most comprehensive analysis to date of post-marketing safety data of tapentadol relating to the topics overall safety, dose administration above approved dosages, administration during pregnancy, serotonin syndrome, respiratory depression, and convulsion following its administration for a wide range of pain conditions. These data collected from spontaneous reports by healthcare and non-healthcare professionals were put in the context of safety information known from interventional and non-interventional trials as well as preclinical findings. It should, however, be noted that underreporting as well as the typically incomplete case information in the spontaneous setting is an important limitation of this research.
Clinical trials have shown good tolerability of tapentadol in pain management for a treatment period of up to 2 years [9]; the most frequently observed adverse events (more than $10 \%$ of trial subjects) were nausea, dizziness, constipation, headache, and somnolence [46]. Head-to-head comparisons with the classical opioids oxycodone and oxycodone/naloxone have demonstrated a better tolerability profile for tapentadol, in particular regarding gastrointestinal side effects [46-48] which is likely due to the NRI contribution to the analgesic effect of tapentadol allowing for reduced $\mu$ opioid receptor activation to reach efficacy comparable to classical strong opioid analgesics.

Opioid typical gastrointestinal and CNS-related side effects were the most common ADRs stated in post-marketing spontaneous reports, whereas noradrenergic side effects did not seem to be prominent. Nausea and dizziness accounted for $4.9 \%$ and $3.6 \%$ of all included ADRs, respectively. The collected data are in line with the clinical trial results; they did not reveal major risks and thus do not change the overall safety profile identified for tapentadol.

Many elderly patients are affected by (often chronic) pain. When treating these patients, frequent comorbidities requiring a number of different medications (polypharmacy) and thus increasing the risk of drug-drug interactions and side effects need to be considered in the choice of pain medication. From pharmacokinetic studies during the development of tapentadol it was known that tapentadol has a low potential for pharmacokinetic drug-drug interaction and clinically relevant interactions are unlikely to occur $[6,7]$. In our comparison of elderly and younger subjects ( $<65$ years of age) in the clinical and post-marketing databases, gastrointestinal and CNS-related drug reactions were the most commonly observed adverse effects in both groups. Clinical comparisons to oxycodone showed significantly better gastrointestinal tolerability in elderly patients for tapentadol both for the IR and PR formulations $[47,49]$. Overall, tapentadol was well tolerated in elderly patients, no new risks were identified for this age group and we can only speculate that the observed differences concerning constipation, confusional state, nausea, and 
headache are more likely age-related than tapentadol related.

Pain treatment with all analgesics and in particular with substances with an opioid mechanism of action has shown that not all patients respond equally to treatment: some patients might require dose adaptations or a switch to a different analgesic to achieve or maintain pain relief. Classically, this is reflected in spontaneous ADR reports of lack of efficacy. For tapentadol treatment, "drug ineffectiveness" was documented in $6.9 \%$ of the spontaneously reported cases.

In clinical practice, many of the reported cases of tapentadol intake exceeding the recommended dosage occurred during pain treatment in order to achieve stronger analgesia; in some cases, abuse and suicide attempts were the reason for a higher tapentadol intake, mostly in combination with other medications. In some cases, exact dosing information was not available; overall, dosages did not greatly exceed recommended tapentadol dosages for many patients. Medical consequences of higher than recommended dosages during routine clinical practice were mainly gastrointestinal, psychiatric, and nervous system disorders (documented in the NIT database); in the spontaneous report database, somnolence $(1.4 \%)$, coma $(1.5 \%)$, and respiratory depression (1.4\%) were also noted. None of the 16 reported fatalities could be clearly attributed to a higher than recommended tapentadol intake. The data collected from tapentadol usage above the currently recommended dosages did not provide evidence for an increased risk for tapentadol use outside the therapeutic window.

Data about the safety of prenatal tapentadol exposure are limited, as pregnant subjects were excluded from clinical trials for ethical reasons and generally did not receive the medication in routine clinical practice because of recommendations to only use tapentadol in pregnancy "if the potential benefit justifies the potential risk to the fetus" [26]. The available clinical and post-marketing data show mostly healthy, normal term babies and no incidence of birth defects following exposure to tapentadol. It should, however, be noted that pregnancy outcome was not reported for the majority of post- marketing cases which limits further conclusions about the potential risk.

Because of the preclinical findings, serotonin syndrome is a theoretical risk of tapentadol treatment; the isolated reports of serotonin syndrome in a temporal connection of the combined use of tapentadol and serotonergic substances are therefore included in the product information [26]. The diagnosis of serotonin syndrome requires a typical constellation of symptoms (clonus, agitation, diaphoresis, tremor, hyperreflexia, hypertonia, and temperature greater than $38^{\circ} \mathrm{C}$ ) [34]. Some of these clinical features are unspecific, others belong to the side effect spectrum of tapentadol, and it is challenging to reach the correct diagnosis. Physicians might mistakenly diagnose the occurrence of some of these symptoms in a patient as serotonin syndrome even if the typical constellation of symptoms is not present [32]. This could be triggered by the list of possible symptoms described in the product information $[26,50]$. The diagnosis of probable serotonin syndrome presented by Walczyk and colleagues [38] (listed in our publication as case 4 in Table 7), for example, was questioned in two letters to the editor $[51,52]$. In the authors' opinion, symptoms were more consistent with an opioid overdose and opioid withdrawal after naloxone reversal, and evidence that serotonin syndrome occurred was deemed insufficient. Of the 151 post-marketing cases collected in the safety database, only four fulfilled the Hunter diagnostic criteria (for case 4 (Table 7) from the literature [38], this diagnosis was questioned by another author group [51]), and could be robustly diagnosed. These cases did not show a definite association of tapentadol with the occurrence of serotonin syndrome. It should be noted that many of the 151 reports, however, lacked information about symptoms and thus precluded a reasonable evaluation.

There were no cases of serotonin syndrome in our ICT database and the diagnosis of the one case documented in the NIT database is questionable. A retrospective analysis of safety data from 11 randomized, double-blind, placebocontrolled acute and chronic pain phase 3 trials (including both IR and PR tapentadol formulations) investigated potential pharmacodynamic 
interactions with concomitant use of tapentadol and SSRIs and/or SNRIs [53]. Adverse event incidences were compared for tapentadol + SSRI/SNRI $(n=208)$ and placebo + SSRI/ SNRI $(n=137)$. The observed treatment emergent adverse events (TEAEs) were mostly expected from tapentadol product labeling. The analysis did not identify new clinically relevant adverse drugs interactions associated with concomitant use of tapentadol and SSRI/SNRIs. Analyses of data from nine phase $2 / 3$ tapentadol IR trials (2178 subjects) and from nine phase $2 / 3$ tapentadol PR trials (3613 subjects) also did not identify any cases of serotonin syndrome reported as an adverse event [54].

Respiratory depression is an ADR of medications with an opioid mechanism of action. It was rarely reported in clinical tapentadol trials and resulted in trial discontinuation in $15 \%$ of the cases. Courses were mostly mild or moderate without severe outcome. Occurrence in spontaneous reporting was also low; in most spontaneous cases, other confounding factors were present (concomitant CNS medications, risk factors of respiratory depression). A recent trial comparing tapentadol IR and oxycodone IR observed greater respiratory depression under oxycodone than under tapentadol at equianalgesic doses [55]. The authors suggest that differences in respiratory effect may be attributed to the difference of the two medications in their affinity for the $\mu$-opioid receptor.

Clinical tapentadol trials did not permit the inclusion of subjects with a seizure disorder; the administration of tapentadol to patients at risk is therefore not recommended. However, postmarketing exposure including patients at risk of seizures did not identify a risk beyond the existing knowledge. The number of cases with a medical history of convulsion risk factors was small though, potentially because of the warning in the product information [26]. Any interpretation of this result is therefore limited.

\section{CONCLUSIONS}

On the basis of the presented descriptive analysis of post-marketing data, the first years of routine clinical practice experience with tapentadol have confirmed the tolerability profile demonstrated in clinical trials; no new major risks were identified. It should be noted that the limitations of the spontaneous data analyzed are underreporting of adverse reactions and the mostly incomplete information in spontaneous reports. The reporting of expected side effects such as respiratory depression and convulsion was low. The available data did not confirm the theoretical risk of serotonin syndrome. The evaluation of reports on administration of higher than recommended tapentadol doses did not reveal any unexpected side effects. Tapentadol shows a favorable overall safety profile in the treatment of acute and chronic pain.

\section{ACKNOWLEDGEMENTS}

The authors would like to thank the patients, investigators, and study teams involved in the studies included in the ICT and NIT databases as well as all healthcare practitioners and consumers reporting tapentadol safety events. They would also like to thank Jérôme Rapion for statistical analyses, Reinhard-Michael Reichert for the programming of safety data retrievals from the global safety database, and Thomas Tzschentke and Corinne van Dorp for their comments on the preclinical manuscript sections.

Funding. All analyses, article processing charges, and the open access fee were funded by Grünenthal $\mathrm{GmbH}$. All authors had full access to all of the data in this study and take complete responsibility for the integrity of the data and accuracy of the data analysis.

Medical Writing and/or Editorial Assistance. Medical writing support was provided by Elke Grosselindemann and Birgit Brett of Brett Medical Writing. This support was funded by Grünenthal $\mathrm{GmbH}$.

Authorship. All named authors meet the International Committee of Medical Journal Editors (ICMJE) criteria for authorship for this 
manuscript, take responsibility for the integrity of the work as a whole, and have given final approval for the version to be published.

Disclosures. Ariane Stollenwerk is an employee of Grünenthal GmbH. Melanie Sohns is an employee of Grünenthal GmbH. Fabian Heisig is an employee of Grünenthal $\mathrm{GmbH}$. Christian Elling is an employee of Grünenthal $\mathrm{GmbH}$. Detlef von Zabern was an employee of Grünenthal $\mathrm{GmbH}$ at the time of authorship definition. Detlef von Zabern is now a partner of medwiss-extern.

Compliance with Ethics Guidelines. This article is based on previously conducted studies and does not involve any new studies of human or animal subjects performed by any of the authors.

Data Availability. The datasets generated during and/or analyzed during the current study are not publicly available because of data privacy but are available from the corresponding author on reasonable request.

Open Access. This article is distributed under the terms of the Creative Commons Attribution-NonCommercial 4.0 International License (http://creativecommons.org/licenses/ by-nc/4.0/), which permits any noncommercial use, distribution, and reproduction in any medium, provided you give appropriate credit to the original author(s) and the source, provide a link to the Creative Commons license, and indicate if changes were made.

\section{REFERENCES}

1. Kress HG. Tapentadol and its two mechanisms of action: is there a new pharmacological class of centrally-acting analgesics on the horizon? Eur J Pain. 2010;14:781-3.

2. Tzschentke TM, Christoph T, Kögel BY. The muopioid receptor agonist/noradrenaline reuptake inhibition (MOR-NRI) concept in analgesia: the case of tapentadol. CNS Drugs. 2014;28:319-29.
3. Schröder $\mathrm{W}$, Tzschentke TM, Terlinden $\mathrm{R}$, et al. Synergistic interaction between the two mechanisms of action of tapentadol in analgesia. J Pharmacol Exp Ther. 2011;337:312-20.

4. Terlinden R, Kogel BY, Englberger W, Tzschentke TM. In vitro and in vivo characterization of tapentadol metabolites. Methods Find Exp Clin Pharmacol. 2010;32:31-8.

5. Terlinden R, Ossig J, Fliegert F, Lange C, Göhler K. Absorption, metabolism, and excretion of ${ }^{14} \mathrm{C}$-labeled tapentadol $\mathrm{HCl}$ in healthy male subjects. Eur J Drug Metab Pharmacokinet. 2007;32:163-9.

6. Kneip C, Terlinden R, Beier H, Chen G. Investigations into the drug-drug interaction potential of tapentadol in human liver microsomes and fresh human hepatocytes. Drug Metab Lett. 2008;2:67-75.

7. Smit JW, Oh C, Rengelshausen J, et al. Effects of acetaminophen, naproxen, and acetylsalicylic acid on tapentadol pharmacokinetics: results of two randomized, open-label, cross-over, drug-drug interaction studies. Pharmacotherapy. 2010;30:25-34.

8. Xiao JP, Li AL, Feng BM, Ye Y, Wang GJ. Efficacy and safety of tapentadol immediate release assessment in treatment of moderate to severe pain: a systematic review and meta-analysis. Pain Med. 2016. https://doi.org/10.1093/pm/pnw154.

9. Baron R, Eberhart L, Kern KU, et al. Tapentadol prolonged release for chronic pain-a review of clinical trials and five years of routine clinical practice data. Pain Pract. 2016. https://doi.org/10. 1111/papr.12515.

10. Daniels SE, Upmalis D, Okamoto A, Lange C, Haeussler J. A randomized, double-blind, phase III study comparing multiple doses of tapentadol IR, oxycodone IR, and placebo for postoperative (bunionectomy) pain. Curr Med Res Opin. 2009;25:765-76.

11. Daniels S, Casson E, Stegmann JU, et al. A randomized, double-blind, placebo-controlled phase 3 study of the relative efficacy and tolerability of tapentadol IR and oxycodone IR for acute pain. Curr Med Res Opin. 2009;25:1551-61.

12. Vorsanger GJ, Klopfer AM, Xiang J, Benson CJ, Moskovitz BL, Rosenthal NR. Immediate-release tapentadol or oxycodone for treatment of acute postoperative pain after elective arthroscopic shoulder surgery: a randomized, phase IIIb study. J Opioid Manag. 2013;9:281-96.

13. Hartrick C, Van Hove I, Stegmann JU, Oh C, Upmalis D. Efficacy and tolerability of tapentadol immediate release and oxycodone $\mathrm{HCl}$ immediate 
release in patients awaiting primary joint replacement surgery for end-stage joint disease: a 10-day, phase III, randomized, double-blind, active- and placebo-controlled study. Clin Ther. 2009;31:260-71.

14. Hale M, Upmalis D, Okamoto A, Lange C, Rauschkolb C. Tolerability of tapentadol immediate release in patients with lower back pain or osteoarthritis of the hip or knee over 90 days: a randomized, doubleblind study. Curr Med Res Opin. 2009;25:1095-104.

15. Afilalo M, Etropolski MS, Kuperwasser B, et al. Efficacy and safety of tapentadol extended release compared with oxycodone controlled release for the management of moderate to severe chronic pin related to osteoarthritis of the knee. Clin Drug Investig. 2010;30:489-505.

16. Wild JE, Grond S, Kuperwasser B, et al. Long-term safety and tolerability of tapentadol extended release for the management of chronic low back pain or osteoarthritis pain. Pain Pract. 2010;10:416-27.

17. Buynak R, Shapiro DY, Okamoto A, et al. Efficacy and safety of tapentadol extended release for the management of chronic low back pain: results of a prospective, randomized, double-blind, placeboand active-controlled phase III study. Expert Opin Pharmacother. 2010;11:1787-804.

18. Baron R, Martin-Mola E, Müller M, Dubois C, Falke D, Steigerwald I. Effectiveness and safety of tapentadol prolonged release (PR) versus a combination of tapentadol PR and pregabalin for the management of severe, chronic low back pain with a neuropathic component: a randomized, double-blind, phase 3b study. Pain Pract. 2015;15:455-70.

19. Baron R, Likar R, Martin-Mola E, et al. Effectiveness of tapentadol prolonged release (PR) compared with oxycodone/naloxone PR for the management of severe chronic low back pain with a neuropathic component: a randomized, controlled, open-label, phase 3b/4 study. Pain Pract. 2016;16:580-99.

20. Schwartz S, Etropolski M, Shapiro DY, et al. Safety and efficacy of tapentadol ER in patients with painful diabetic peripheral neuropathy: results of a randomized-withdrawal, placebo-controlled trial. Curr Med Res Opin. 2011;27:151-62.

21. Vinik AI, Shapiro DY, Rauschkolb C, et al. A randomized withdrawal, placebo-controlled study evaluating the efficacy and tolerability of tapentadol extended release in patients with chronic, painful diabetic peripheral neuropathy. Diabetes Care. 2014;37:2302-9.

22. Imanaka K, Tominaga $Y$, Etropolski $M$, et al. Efficacy and safety of oral tapentadol extended release in Japanese and Korean patients with moderate to severe, chronic malignant tumor-related pain. Curr Med Res Opin. 2013;29:1399-409.

23. Kress HG, Koch ED, Kosturski H, et al. Tapentadol prolonged release for managing moderate to severe, chronic malignant tumor-related pain. Pain Physician. 2014;17:329-43.

24. Buynak R, Rappaport SA, Rod K, et al. Long-term safety and efficacy of tapentadol extended release following up to 2 years of treatment in patients with moderate to severe, chronic pain: results of an openlabel extension trial. Clin Ther. 2015;37:2420-38.

25. Electronic Medicines Compendium. Palexia film coated tablets. https://www.medicines.org.uk/emc/ medicine/28375. Accessed Feb 15, 2017.

26. Electronic Medicines Compendium. Palexia SR prolonged release tablets. https://www.medicines. org.uk/emc/medicine/28373. Accessed Sept 19, 2016.

27. Tzschentke TM, De Vry J, Terlinden R, et al. Tapentadol hydrochloride. Analgesic mu-opioid receptor agonist noradrenaline reuptake inhibitor. Drugs Future. 2006;31:1053-61.

28. Desai RJ, Huybrechts KF, Hernandez-Diaz S, et al. Exposure to prescription opioid analgesics in utero and risk of neonatal abstinence syndrome: population based cohort study. BMJ. 2015;350:h2102.

29. Yadzy MM, Desai RJ, Brogly SB. Prescription opioids in pregnancy and birth outcomes: a review of the literature. J Pediatr Genet. 2015;4:56-70.

30. Lassen D, Ennis ZN, Damkier P. First-trimester pregnancy exposure to venlafaxine or duloxetine and risk of major congenital malformations: a systematic review. Basic Clin Pharmacol Toxicol. 2016;118:32-6.

31. Bellantuono C, Vargas M, Mandarelli G, Nardi B, Martini MG. The safety of serotonin-noradrenaline reuptake inhibitors (SNRIs) in pregnancy and breastfeeding: a comprehensive review. Hum Psychopharmacol. 2015;30:143-51.

32. Gillman PK. Monoamine oxidase inhibitors, opioid analgesics and serotonin toxicity. $\mathrm{Br} \mathrm{J}$ Anaesth. 2005;95:434-41.

33. Sun-Edelstein C, Tepper SJ, Shapiro RE. Drug-induced serotonin syndrome: a review. Expert Opin Drug Saf. 2008;7:587-96.

34. Dunkley EJC, Isbister GK, Sibbritt D, Dawson AH, Whyte IM. The Hunter serotonin toxicity criteria: simple and accurate diagnostic decision rules for serotonin toxicity. Q J Med. 2003;96:635-42. 
35. Tzschentke TM, Christoph T, Kögel B, et al. (-)$(1 R, 2 R)$-3-(3-Dimethylamino-1-ethyl-2-methyl-propyl)-phenol hydrochloride (tapentadol $\mathrm{HCl}$ ): a novel $\mu$-opioid receptor agonist/norepinephrine reuptake inhibitor with broad-spectrum analgesic properties. J Pharmacol Expert Ther. 2007;323:265-76.

36. Bloms-Funke $\mathrm{P}$, Dremencov E, Cremers TI, Tzschentke TM. Tramadol increases extracellular levels of serotonin and noradrenaline as measured by in vivo microdialysis in the ventral hippocampus of freely-moving rats. Neurosci Lett. 2011;490:191-5.

37. Schröder W, De Vry J, Tzschentke TM, Jahnel U, Christoph T. Differential contribution of opioid and noradrenergic mechanisms of tapentadol in rat models of nociceptive and neuropathic pain. Eur J Pain. 2010:814-21.

38. Walczyk H, Liu CH, Alafris A, Cohen H. Probable tapentadol-associated serotonin syndrome after overdose. Hosp Pharm. 2016;51:320-7.

39. Pattinson KTS. Opioids and the control of respiration. Br J Anaesth. 2008;100:747-58.

40. Lalley PM. Opioidergic and dopaminergic modulation of respiration. Respir Physiol Neurobiol. 2008;164:160-7.

41. Larson SJ, Pestaner J, Prashar SK, Bayard C, Zarwell LW, Pierre-Louis M. Postmortem distribution of tapentadol and $n$-desmethyltapentadol. J Anal Toxicol. 2012;36:440-3.

42. Gutstein HB, Akil H. Opioid analgesics. In: Brunton L, editor. Goodman and Gilman's. The pharmacological basis of therapeutics. 11th ed. Pennsylvania: McGraw-Hill; 2006. p. 547-90.

43. Raffa RB, Stone DJ. Unexceptional seizure potential of tramadol or its enantiomers or metabolites in mice. J Pharmacol Exp Ther. 2008;325:500-6.

44. Gasse C, Derby L, Vasilakis-Scaramozza C, Jick H. Incidence of first-time idiopathic seizures in users of tramadol. Pharmacotherapy. 2000;20:629-34.

45. Food and Drug Administration. Nucynta summary basis for approval. https://www.accessdata.fda.gov/ drugsatfda_docs/nda/2008/022304s000_PharmR_ P5.pdf. Accessed June 15, 2017.

46. Etropolski M, Kuperwasser B, Flügel M, et al. Safety and tolerability of tapentadol extended release in moderate to severe chronic osteoarthritis or low back pain management: pooled analysis of randomized controlled trials. Adv Ther. 2014;31:604-20.

47. Vorsanger G, Xiang J, Biondi D, et al. Post hoc analyses of data from a 90-day clinical trial evaluating the tolerability and efficacy of tapentadol immediate release and oxycodone immediate release for the relief of moderate to severe pain in elderly and nonelderly patients. Pain Res Manag. 2011;16:245-51.

48. Baron R, Jansen JP, Binder A, et al. Tolerability, safety, and quality of life with tapentadol prolonged release (PR) compared with oxycodone/naloxone $\mathrm{PR}$ in patients with severe chronic low back pain with a neuropathic component: a randomized, controlled, open-label, phase 3b/4 trial. Pain Pract. 2016;16:600-19.

49. Biondi DM, Xiang J, Häufel T, Etropolski M, Moskovitz B. Tolerability and efficacy of tapentadol extended release in elderly patients $\geq 75$ years of age with chronic osteoarthritis knee or low back pain. J Opioid Manag. 2015;11:393-403.

50. Janssen Pharmaceuticals. NUCYNTA ER-tapentadol hydrochloride tablet, film coated, extended release. https://www.nucynta.com/sites/default/files/pdf/ nucyntaer-pi_0.pdf. Accessed Mar 6, 2017.

51. Mullins ME, Dribben WH. Comment on tapentadol and serotonin syndrome. Hosp Pharm. 2017;52:246-7.

52. Russo M, Santarelli D, Isbister G. Comment on "probable tapentadol-associated serotonon syndrome after overdose". Hosp Pharm. 2017;52:248.

53. Brett V, Sikes C, Xiang J, Oh C, Biondi D. Post hoc analysis of pooled safety data from eleven phase 3 clinical trials to identify potential pharmacodynamic drug interactions between tapentadol and SSRIs/SNRIs. J Pharm Pract. 2012;25:265.

54. Schmidt P. Safety information on serotonin syndrome for tapentadol IR and tapentadol ER administration (Abstract poster 212). http://www. painmed.org/2016posters/abstract-212/. Accessed Sept 26, 2016.

55. van der Schrier R, Jonkman K, van Velzen M, et al. An experimental study comparing the respiratory effects of tapentadol and oxycodone in healthy volunteers. Br J Anaesth 2017;119(6):1169. 